\title{
Consensus of Multiagent Networks with Intermittent Interaction and Directed Topology
}

\author{
Li Xiao \\ Department of Mathematics and Information Engineering, Chongqing University of Education, Chongqing 400067, China \\ Correspondence should be addressed to Li Xiao; xsxiaoli@163.com
}

Received 24 May 2014; Revised 22 July 2014; Accepted 22 July 2014; Published 12 August 2014

Academic Editor: Chuandong Li

Copyright (C) 2014 Li Xiao. This is an open access article distributed under the Creative Commons Attribution License, which permits unrestricted use, distribution, and reproduction in any medium, provided the original work is properly cited.

Intermittent interaction control is introduced to solve the consensus problem for second-order multiagent networks due to the limited sensing abilities and environmental changes periodically. And, we get some sufficient conditions for the agents to reach consensus with linear protocol from the theoretical findings by using the Lyapunov control approach. Finally, the validity of the theoretical results is validated through the numerical example.

\section{Introduction}

The problem of coordinating the motion of multiagent networks has attracted increasing attention. Research on multiagent coordinated control problems not only helps in better understanding the general mechanisms and interconnection rules of natural collective phenomena but also carries out benefits in many practical applications of networked cyberphysical systems, such as tracking [1], flocking [2,3], and formation [4]. Consensus, along with stability [5] and bifurcation [6], is a fundamental phenomenon in nature [7]. Roughly speaking, consensus means that all agents in network will converge to some common state by negotiating with their neighbors. A consensus algorithm is an interaction rule on how agents update their states.

To realize consensus, many effective approaches were proposed [8-10]. Since the network can be regarded as a graph, the issues can be depicted by the graph theory. The recent approaches concentrate on matrix analysis [11], convex analysis [12, 13], and graph theory [14]. The concept of spanning tree especially is widely used to describe the communicability between agents in networks that can guarantee the consensus [15]. For more consensus problems, the reader may refer to [16-21] and the references therein.

As we know, sometimes only the intermittent states of its neighbors can be obtained by the agents to the transmission capacity, communication cost, sensing abilities, and the environmental changes. To decrease the control cost, only the intermittent states of its neighbors are obtained [22]. This is mainly because such networks are constrained by the following operational characteristics: (i) they may not have a centralized entity for facilitating computation, communication, and timesynchronization, (ii) the network topology may not be completely known to the nodes of the network, and (iii) in the case of sensor networks, the computational power and energy resources may be very limited. Inspired by the above consideration, the goal in this setting is to design algorithms by exploiting partial state sampling at each node; it is possible to reduce the amount of data which needs to be transmitted in networks, thereby saving bandwidth and energy, extending the network lifetime, and reducing latency. Also, the linear local interaction protocol can guarantee the linear nature of distributed multiagent networks in real world and linear algorithm is simple and easy to implement so as to be widely used in practical engineering especially in the limited transmission environment. Using the Lyapunov control approach, some sententious conditions are obtained in this paper for reaching consensus in multiagent networks.

The rest of this paper is organized as follows. In Section 2, some preliminaries on the graph theory and the model formulation are given. The main results are established in Section 3. In Section 4, a numerical example is simulated to verify the theoretical analysis. Concise conclusions are finally drawn in Section 5. 


\section{Preliminaries and Model}

2.1. Graph Theory. In this subsection, some basic concepts and result of algebraic grapy theory are introduced. Suppose that information exchange among agents in multiagent networks can be modeled by an interaction digraph.

Let $g=(V, \mathcal{\varepsilon}, A)$ denote a directed graph with the set of nodes $V=\{1,2, \ldots, N\}$, where $\varepsilon \subseteq V \times V$ represents the edge set and $A=\left(a_{i j}\right)_{N \times N}$ is the adjacency matrix with nonnegative elements $a_{i j}$. A directed edge $\varepsilon_{i j}$ in the network $g$ is denoted by the ordered pair of nodes $(i, j)$, where $i$ is the receiver and $j$ is the sender, which means that node $i$ can receive information from node $j$. We always assume that there is no self-loop in network $g$. An adjacency matrix $A$ of a directed graph can be defined such that $a_{i j}$ is a nonnegative element if $\varepsilon_{i j} \in \varepsilon$, while $a_{i j}=0$ if $\varepsilon_{i j} \notin \varepsilon$. The set of neighbors of node $i$ is denoted by $N_{i}=\{j \in V:(i, j) \in \varepsilon\}$. A sequence of edges of the form $\left(i, j_{1}\right),\left(j_{1}, j_{2}\right), \ldots,\left(j_{m}, j\right) \in \varepsilon$ composes a directed path beginning with $i$ and ending with $j$ in the directed graph $g$ with distinct nodes $j_{k}, k=1,2, \ldots, m$, which means the node $j$ is reachable from node $i$. A directed graph is strongly connected if for any distinct nodes $i$ and $j$, there exists a directed path from node $i$ to node $j$. A directed graph has a directed spanning tree if there exists at least one node called root which has a directed path to all the other nodes [16]. Let (generally nonsymmetrical) Laplacian matrix $L=\left(l_{i j}\right)_{N \times N}$ associated with directed network $g$ be defined by

$$
l_{i j}= \begin{cases}\sum_{k=1, k \neq i}^{N} a_{i k}, & i=j, \\ -a_{i j}, & i \neq j,\end{cases}
$$

which ensure the diffusion property $\sum_{j=1}^{N} l_{i j}=0$. Suppose $L$ is irreducible. Then, $L 1_{N}=0_{N}$ and there is a positive vector $\xi=\left(\xi_{1}, \xi_{2}, \ldots, \xi_{N}\right)^{T}$ satisfying $\xi^{T} L=0_{N}$ and $\xi^{T} \mathbf{1}_{N}=1$. In addition, there exists a positive definite diagonal matrix $\Xi=$ $\operatorname{diag}\left(\xi_{1}, \xi_{2}, \ldots, \xi_{N}\right)$ such that $\bar{L}=\left(\Xi L+L^{T} \Xi\right) / 2$ is symmetric and $\sum_{j=1}^{N} \bar{L}_{i j}=\sum_{j=1}^{N} \bar{L}_{j i}=0$ for all $i=1,2, \ldots, N$ [18].

For simplicity, some mathematical notations are used throughout this paper. $I_{n}\left(O_{n}\right)$ denotes the identity (zero) matrix with $n$ dimensions. Let $\mathbf{1}_{n}\left(\mathbf{0}_{n}\right)$ be the vector with all $n$ elements being $1(0) . R^{n}$ is the $n$-dimensional real vector space. The notation $\otimes$ denotes the Kronecker product.

2.2. Model Description. The discretization process of a continuous-time system cannot entirely preserve the dynamics of the continuous-time part even small sampling period is adopted. So, we consider the following second-order multiagent networks of $N$ agents in [19] with intermittent measurements. The $i$ th agent in the directed network $g$ is governed by double-integrator dynamics

$$
\begin{gathered}
\dot{x}_{i}(t)=v_{i}(t), \\
\dot{v}_{i}(t)=d(t)\left(\sum_{j=1, j \neq i}^{N} a_{i j}\left(x_{j}(t)-x_{i}(t)\right)\right. \\
\left.+\kappa \sum_{j=1, j \neq i}^{N} a_{i j}\left(v_{j}(t)-v_{i}(t)\right)\right), \\
i=1,2, \ldots N,
\end{gathered}
$$

where $x_{i}(t) \in R^{n}$ and $v_{i}(t) \in R^{n}$ are the position and velocity states of the $i$ th agent, respectively. $\kappa$ denotes the coupling strengths. $d(t)$ denotes the intermittent control as follows:

$$
d(t)= \begin{cases}1, & t_{0}+k \omega<t \leq t_{0}+k \omega+\delta, \\ 0, & t_{0}+k \omega+\delta<t \leq t_{0}+(k+1) \omega,\end{cases}
$$

where $\omega>0$ is the control period and $\delta>0$ is called the control width.

Equivalently, model (2) can be rewritten as follows:

$$
\begin{gathered}
\dot{x}_{i}(t)=v_{i}(t), \\
\dot{v}_{i}(t)=-d(t)\left(\sum_{j=1}^{N} l_{i j} x_{j}(t)+\kappa \sum_{j=1}^{N} l_{i j} v_{j}(t)\right), \\
i=1,2, \ldots, N .
\end{gathered}
$$

In this paper, our goal is to design suitable $\omega, \delta$ such that the network reaches consensus. In the following we present the following lemma and definitions.

Lemma 1 (see [23]). Suppose that $M \in R^{n \times n}$ is positive definite and $N \in R^{n \times n}$ is symmetric. Then $\forall x \in R^{n}$, and the following inequality holds:

$$
\lambda_{\max }\left(M^{-1} N\right) x^{T} M x \geq x^{T} N x \geq \lambda_{\min }\left(M^{-1} N\right) x^{T} M x .
$$

Definition 2 (see [18]). Let $\xi, \Xi$, and $\bar{L}$ be defined as in Section 2.1. For a strongly connected network with Laplacian matrix $L$, let

$$
a(L)=\min _{x^{T} \xi=0, x \neq 0} \frac{x^{T} \bar{L} x}{x^{T} \Xi x}, \quad b(L)=\max _{x^{T} \xi=0, x \neq 0} \frac{x^{T} \bar{L} x}{x^{T} \Xi x} .
$$

Definition 3. Periodic intermittent consensus in the secondorder multiagent networks (2) is said to be achieved if, for any initial conditions,

$$
\begin{gathered}
\lim _{t \rightarrow \infty}\left\|x_{i}(t)-x_{j}(t)\right\|=0, \\
\lim _{t \rightarrow \infty}\left\|v_{i}(t)-v_{j}(t)\right\|=0, \quad \forall i, j=1,2, \ldots, N .
\end{gathered}
$$

\section{Main Results}

In this section, we will focus on consensus analysis of secondorder multiagent networks via intermittent control in the strongly connected networks, simply for that a matrix $G$ is irreducible if and only if its corresponding system $G$ is strongly connected [24].

Let $\bar{x}=\sum_{k=1}^{N} \xi_{k} x_{k}(t), \bar{v}=\sum_{k=1}^{N} \xi_{k} v_{k}(t)$ represent the average position and velocity of agent. Naturally, $\widehat{x}_{i}(t)=$ $x_{i}(t)-\bar{x}$ and $\widehat{v}_{i}(t)=v_{i}(t)-\bar{v}$ represent the position and velocity vectors relative to the average position and velocity of the agents in network. Then the error dynamical system can be rewritten in a compact matrix form as

$$
\dot{y}(t)=\left(\widehat{L} \otimes I_{n}\right) y(t),
$$

where $\widehat{L}=\left(\begin{array}{cc}0_{N} & I_{N} \\ -\alpha d(t) L & -\beta d(t) L\end{array}\right)$. 
Theorem 4. Suppose that the agent network is strongly connected; then the linear consensus in the multiagent networks (2) via periodic intermittent interaction is achieved if the following conditions are satisfied:

(1) $a(L)>1 / \kappa^{2}$,

(2) $\gamma(\omega-\delta)+\eta \delta<0, \gamma>0, \eta<0$,

where

$$
\begin{gathered}
\gamma=2 \lambda_{\max }\left(B^{-1} E\right)>0, \quad \eta=2 \lambda_{\max }(C) / \lambda_{\max }(D)<0, \\
B=\left(\begin{array}{cc}
2 \kappa a(L) \Xi & \Xi \\
\Xi & \kappa \Xi
\end{array}\right), \quad C=\left(\begin{array}{cc}
-a(L) \Xi & O_{N} \\
O_{N} & \Xi-\kappa^{2} a(L) \Xi
\end{array}\right), \\
D=\left(\begin{array}{cc}
2 \kappa b(L) \Xi & \Xi \\
\Xi & \kappa \Xi
\end{array}\right), \\
E=\left(\begin{array}{cc}
O_{N} & (\kappa / 2)\left(\Xi L+L^{T} \Xi\right) \\
(\kappa / 2)\left(\Xi L+L^{T} \Xi\right) & \Xi
\end{array}\right) .
\end{gathered}
$$

Proof. The potential Lyapunov function is defined to be

$$
V(t)=\frac{1}{2} y^{T}(t)\left(\Omega \otimes I_{n}\right) y(t)
$$

where $\Omega=\left(\begin{array}{cc}2 \kappa \bar{L} & \Xi \\ \kappa \Xi\end{array}\right)$. Computing by the Definition 2, one obtains

$$
\begin{aligned}
& V(t)= \frac{1}{2} \widehat{x}^{T}(t)\left((2 \kappa \bar{L}) \otimes I_{n}\right) \hat{x}(t)+\frac{1}{2} \widehat{x}^{T}(t)\left((\Xi) \otimes I_{n}\right) \widehat{v}(t) \\
&+\frac{1}{2} \widehat{v}^{T}(t)\left((\Xi) \otimes I_{n}\right) \hat{x}(t)+\frac{1}{2} \widehat{v}^{T}(t)\left((\kappa \Xi) \otimes I_{n}\right) \widehat{v}(t) \\
& \geq \frac{1}{2} y^{T}(t)\left(B \otimes I_{n}\right) y(t), \\
& V(t) \leq \frac{1}{2} y^{T}(t)\left(D \otimes I_{n}\right) y(t),
\end{aligned}
$$

where $B=\left(\begin{array}{cc}2 \kappa a(L) \Xi & \Xi \\ \Xi & \kappa \Xi\end{array}\right), D=\left(\begin{array}{cc}2 \kappa b(L) \Xi & \Xi \\ \Xi & \kappa \Xi\end{array}\right)$.

$B>0$ is equivalent to $\Xi>0$ and $2 \kappa a(L) \Xi-(1 / \kappa) \Xi>0$ by Schur Complement Lemma. And it is clear that $a(L)>1 / 2 \kappa^{2}$; thus $B>0$. So $V(t) \geq 0$ and $V(t)=0$ if and only if $y=0$.

Because the control gain $d(t)$ works intermittently by the control period and the control width, the consensus is discussed in the two different intervals, respectively.

Firstly, for $t_{0}+k \omega<t \leq t_{0}+k \omega+\delta$ and $\widehat{L}=\left(\begin{array}{cc}0_{N} & I_{N} \\ -L & -\kappa L\end{array}\right)$, taking the derivative of $V(t)$ along the trajectories of $(8)$, it can be obtained that

$$
\dot{V}(t)=\frac{1}{2} y^{T}(t)\left[\left(\Omega \widehat{L}+\widehat{L}^{T} \Omega\right) \otimes I_{n}\right] y(t) .
$$

In addition,

$$
\begin{aligned}
\Omega \widehat{L} & =\left(\begin{array}{cc}
2 \kappa \bar{L} & \Xi \\
\Xi & \kappa \Xi
\end{array}\right)\left(\begin{array}{cc}
O_{N} & I_{N} \\
-d(t) L & -\kappa d(t) L
\end{array}\right) \\
& =\left(\begin{array}{cc}
-\Xi L & 2 \kappa \bar{L}-\kappa \Xi L \\
-\kappa \Xi L & \Xi-\kappa^{2} \Xi L
\end{array}\right), \\
\widehat{L}^{T} \Omega & =\left(\begin{array}{cc}
-L^{T} \Xi & -\kappa L^{T} \Xi \\
2 \kappa \bar{L}-\kappa L^{T} \Xi & \Xi-\kappa^{2} L^{T} \Xi
\end{array}\right) .
\end{aligned}
$$

Thus, one obtains that

$$
\frac{\Omega \widehat{L}+\widehat{L}^{T} \Omega}{2}=\left(\begin{array}{cc}
-\bar{L} & O_{N} \\
O_{N} & \Xi-\kappa^{2} \bar{L}
\end{array}\right) .
$$

Therefore, from (12) to (14), one obtains

$$
\dot{V}(t) \leq\|y\|^{T} C\|y\|,
$$

where $C=\left(\begin{array}{cc}-a(L) \Xi & O_{N} \\ O_{N} & \Xi-\kappa^{2} a(L) \Xi\end{array}\right)$.

Using $a(L)>1 / \kappa^{2}$ in condition (2), $C$ is a negativedefinite matrix.

Thus,

$$
\dot{V}(t) \leq \lambda_{\max }(C) y^{T}(t) y(t), \quad\left(\lambda_{\max }(C)<0\right) .
$$

And, on the other hand,

$$
\begin{aligned}
V(t) & =\frac{1}{2} y^{T}(t)\left(\Omega \otimes I_{n}\right) y(t) \leq \frac{1}{2} y^{T}(t)\left(D \otimes I_{n}\right) y(t) \\
& \leq \frac{1}{2} \lambda_{\max }(D) y^{T}(t) y(t) .
\end{aligned}
$$

Consequently,

$$
\begin{array}{r}
\dot{V}(t) \leq \eta V(t), \quad \eta=\frac{2 \lambda_{\max }(C)}{\lambda_{\max }(D)}<0, \\
t_{0}+k \omega<t \leq t_{0}+k \omega+\delta .
\end{array}
$$

Thus, we can obtain

$$
V(t) \leq V\left(t_{0}+k \omega\right) e^{\eta\left(t-t_{0}-k \omega\right)}, \quad t_{0}+k \omega<t \leq t_{0}+k \omega+\delta .
$$

Then let us consider the period $t_{0}+k \omega+\delta<t \leq t_{0}+(k+$ 1) $\omega$. For $\widehat{L}=\left(\begin{array}{ll}O_{N} & I_{N} \\ O_{N} & O_{N}\end{array}\right)$, we can derive

$$
\begin{aligned}
\dot{V}(t) & =y^{T}(t)\left(\Omega \otimes I_{n}\right)\left[\left(\widehat{L} \otimes I_{n}\right) y(t)\right] \\
& =\frac{1}{2} y^{T}(t)\left[\left(\Omega \widehat{L}+\widehat{L}^{T} \Omega\right) \otimes I_{n}\right] y(t),
\end{aligned}
$$

where

$$
\begin{gathered}
\Omega \widehat{L}=\left(\begin{array}{cc}
2 \kappa \bar{L} & \Xi \\
\Xi & \kappa \Xi
\end{array}\right)\left(\begin{array}{ll}
O_{N} & I_{N} \\
O_{N} & O_{N}
\end{array}\right)=\left(\begin{array}{cc}
O_{N} & 2 \kappa \bar{L}-\kappa \Xi L \\
O_{N} & \Xi
\end{array}\right), \\
\widehat{L}^{T} \Omega=\left(\begin{array}{cc}
O_{N} & O_{N} \\
2 \kappa \bar{L}^{T} & \alpha \Xi
\end{array}\right) .
\end{gathered}
$$


Then,

$$
\frac{\Omega \widehat{L}+\widehat{L}^{T} \Omega}{2}=\left(\begin{array}{cc}
O_{N} & \kappa \bar{L} \\
\kappa \bar{L}^{T} & \Xi
\end{array}\right) .
$$

Based on the above, one obtains

$$
\dot{V}(t) \leq y^{T}(t) E y(t),
$$

where $E=\left(\begin{array}{cc}O_{N} & (\kappa / 2)\left(\Xi L+L^{T} \Xi\right) \\ (\kappa / 2)\left(\Xi L+L^{T} \Xi\right) & \Xi\end{array}\right)$.

From Lemma 1, we obtain

$$
\begin{aligned}
\dot{V}(t) & \leq y^{T}(t) E y(t) \leq \lambda_{\max }\left(B^{-1} E\right) y^{T}(t) B y(t) \\
& \leq 2 \lambda_{\max }\left(B^{-1} E\right) V(t) .
\end{aligned}
$$

We set $\gamma=2 \lambda_{\text {max }}\left(B^{-1} E\right)>0$, so as to obtain

$$
\begin{array}{r}
V(t) \leq V\left(t_{0}+k \omega+\delta\right) e^{\gamma\left(t-t_{0}-k \omega-\delta\right)}, \\
t_{0}+k \omega+\delta<t \leq t_{0}+(k+1) \omega .
\end{array}
$$

Now, we can obtain from (19) and (25) that

$$
\begin{aligned}
V\left(t_{0}+(k+1) \omega\right) & \leq V\left(t_{0}+k \omega+\delta\right) e^{\gamma(\omega-\delta)} \\
& \leq V\left(t_{0}+k \omega\right) e^{\gamma(\omega-\delta)+\eta \delta} \\
& \leq \cdots \leq V\left(t_{0}\right) e^{[\gamma(\omega-\delta)+\eta \delta](n+1)} .
\end{aligned}
$$

It is clear that there is a constant $n_{0} \geq 0$ satisfying $t_{0}+n_{0} \omega<$ $t \leq t_{0}+\left(n_{0}+1\right) \omega$ for any $t>t_{0}$. Thus we get that for $t_{0}+n_{0} \omega<$ $t \leq t_{0}+n_{0} \omega+\delta$,

$$
\begin{aligned}
V(t) & \leq V\left(t_{0}\right) e^{\eta\left(t-t_{0}-n_{0} \omega\right)} e^{[\gamma(\omega-\delta)+\eta \delta] n_{0}} \\
& \leq V\left(t_{0}\right) e^{[\gamma(\omega-\delta)+\eta \delta] n_{0}} \\
& =V\left(t_{0}\right) e^{-[\gamma(\omega-\delta)+\eta \delta]} e^{[\gamma(\omega-\delta)+\eta \delta]\left(n_{0}+1\right)} \\
& \leq V\left(t_{0}\right) e^{-[\gamma(\omega-\delta)+\eta \delta]} e^{(-(\gamma(\omega-\delta)+\eta \delta) / \omega) t_{0}} e^{((\gamma(\omega-\delta)+\eta \delta) / \omega) t}
\end{aligned}
$$

and for $t_{0}+n_{0} \omega+\delta<t \leq t_{0}+\left(n_{0}+1\right) \omega$,

$$
\begin{aligned}
V(t) & \leq V\left(t_{0}\right) e^{\gamma\left(t-t_{0}-n_{0} \omega-\delta\right)} e^{[\gamma(\omega-\delta)+\eta \delta] n_{0}} \\
& \leq V\left(t_{0}\right) e^{((\gamma(\omega-\delta)+\eta \delta) / \omega)\left[\omega\left(n_{0}+1\right)+t_{0}-t_{0}\right]} \\
& \leq V\left(t_{0}\right) e^{-((\gamma(\omega-\delta)+\eta \delta) / \omega) t_{0}} e^{((\gamma(\omega-\delta)+\eta \delta) / \omega) t} .
\end{aligned}
$$

Hence, let $K=V\left(t_{0}\right) e^{-[\gamma(\omega-\delta)+\eta \delta]} e^{-((\gamma(\omega-\delta)+\eta \delta) / \omega) t_{0}}$, and we can conclude the following from the above analysis:

$$
V(t) \leq K e^{((\gamma(\omega-\delta)+\eta \delta) / \omega) t}, \quad t>t_{0}
$$

which means that the states of agents can achieve consensus. The proof is complete.

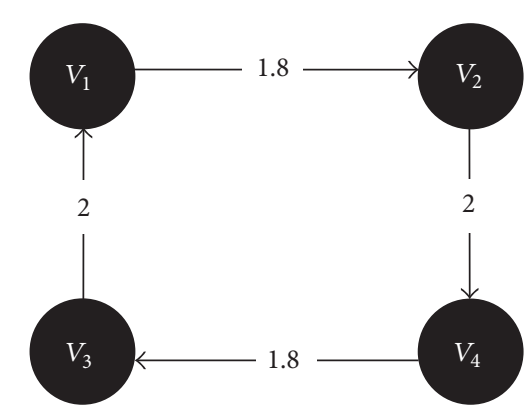

FIGURE 1: The directed interaction topology of multiagent networks.

\section{Numerical Simulations}

A multiagent network of four agents is considered as the simulation example. The multiagent network topology is described by a directed network $g$ shown in Figure 1. It can be seen that the network is strongly connected.

Let $\kappa=2$ and $n=3$. With simple calculations, we obtain the $a(L)=1.8>1 / \kappa^{2}, \eta=-2.1204$, and $\gamma=3.5980$. From condition (3), we obtain $\delta / \omega>0.6292$. So if we set $\delta=0.07$ and $\omega=0.1$, second-order consensus can be achieved in system (2). The initial position and velocity values of agents are $x_{1}=(3,1,-3)^{T}, x_{2}=(6,2,-6)^{T}, x_{3}=(-5,3,-9)^{T}$, $x_{4}=(9,4,-12)^{T}, v_{1}=(2,3,-2)^{T}, v_{2}=(-5,6,3)^{T}, v_{3}=$ $(1,-4,2)^{T}$, and $v_{4}(3,4,-5)^{T}$, respectively. Figure 2 shows the linear consensus of position and velocity states of four agents with intermittent control.

\section{Conclusions}

In this paper, we have considered the linear consensus of multiagent networks with periodic intermittent interaction and directed topology. We choose to show the consensus with linear local interaction protocols, partly for simplifying the problem. On the other hand, it is simple and easy to implement so as to be widely used in practical engineering. The tools from algebraic graph theory, matrix theory, and Lyapunov control approach have been adopted. It is shown that the consensus is determined commonly by the general algebraic connectivity, control period, and control width. And the states of agents converge exponentially.

\section{Conflict of Interests}

The author declares that there is no conflict of interests regarding the publication of this paper.

\section{Acknowledgments}

The author sincerely thanks Junjie Bao (Department of Mathematics and Information Engineering, Chongqing University of Education) for his helpful work that led to the present version. And the work was supported by the Foundation 


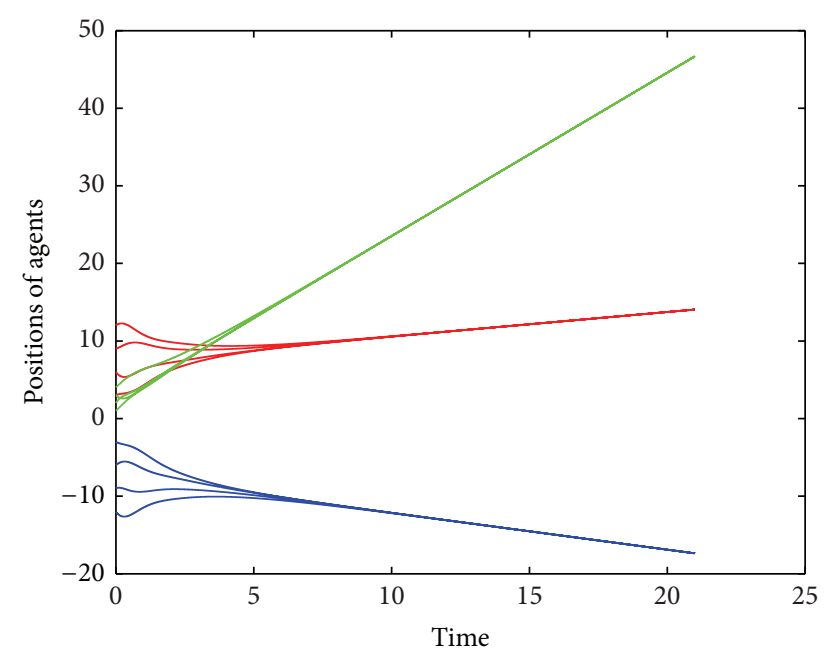

(a)

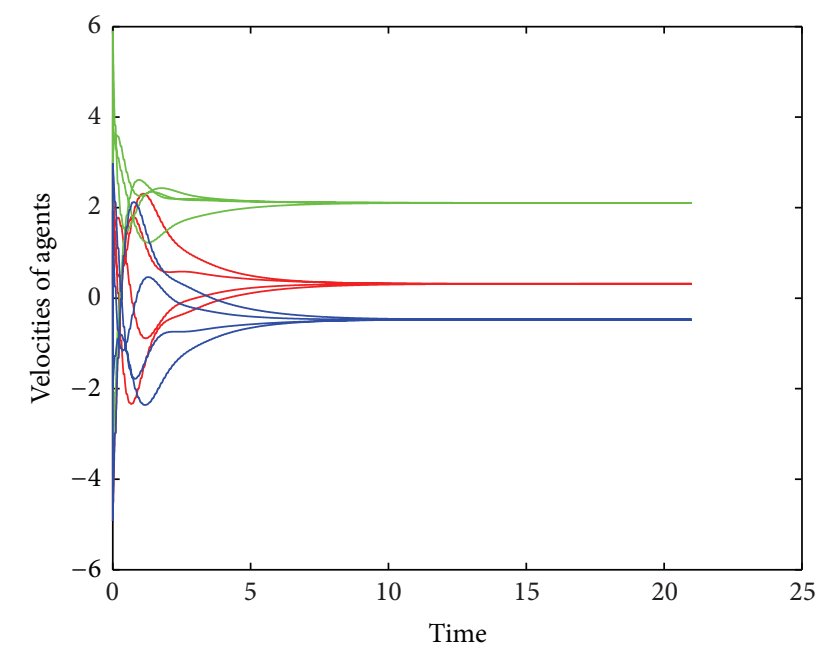

(b)

FIGURE 2: Position and velocity states of four agents in the network.

Project of CQCSTC (no. cstc2014jcyjA40041), the National Natural Science Foundation of China (no. 60973114), and the Foundation of Chongqing University of Education (no. KY201318B).

\section{References}

[1] G. Hu, "Robust consensus tracking of a class of second-order multi-agent dynamic systems," Systems and Control Letters, vol. 61, no. 1, pp. 134-142, 2012.

[2] H. G. Tanner, A. Jadbabaie, and G. J. Pappas, "Flocking in fixed and switching networks," IEEE Transacitons on Automatic Control, vol. 52, no. 5, pp. 863-868, 2007.

[3] H. Zhang, C. Zhai, and Z. Chen, "A general alignment repulsion algorithm for flocking of multi-agent systems," IEEE Transactions on Automatic Control, vol. 56, no. 2, pp. 430-435, 2011.

[4] P. Lin and Y. Jia, "Distributed rotating formation control of multi-agent systems," Systems \& Control Letters, vol. 59, no. 10, pp. 587-595, 2010.
[5] C. Li, C. Li, X. Liao, and T. Huang, "Impulsive effects on stability of high-order BAM neural networks with time delays," Neurocomputing, vol. 74, no. 10, pp. 1541-1550, 2011.

[6] X. He, C. Li, and Y. Shu, "Bogdanov-Takens bifurcation in a single inertial neuron model with delay," Neurocomputing, vol. 89, pp. 193-201, 2012.

[7] C. W. Wu and L. O. Chua, "Synchronization in an array of linearly coupled dynamical systems," IEEE Transactions on Circuits and Systems, vol. 42, no. 8, pp. 430-447, 1995.

[8] J. Hu and Y. Hong, "Leader-following coordination of multiagent systems with coupling time delays," Physica A: Statistical Mechanics and its Applications, vol. 374, no. 2, pp. 853-863, 2007.

[9] W. Yu, G. Chen, Z. Wang, and W. Yang, "Distributed consensus filtering in sensor networks," IEEE Transactions on Systems, Man, and Cybernetics B, vol. 39, no. 6, pp. 1568-1577, 2009.

[10] A. K. Das, R. Fierro, V. Kumar, J. P. Ostrowski, J. Spletzer, and C. J. Taylor, "A vision-based formation control framework," IEEE Transactions on Robotics and Automation, vol. 18, no. 5, pp. 813825, 2002.

[11] Y. Chen, J. Lü, F. Han, and X. Yu, "On the cluster consensus of discrete-time multi-agent systems," Systems and Control Letters, vol. 60 , no. 7, pp. 517-523, 2011.

[12] H. Wang, X. Liao, T. Huang, and C. Li, "Cooperative distributed optimization in multi-agent networks with delays," IEEE Transactions on Systems, Man, and Cybernetics: Systems, no. 99, p. 1, 2014.

[13] L. Moreau, "Stability of multiagent systems with time-dependent communication links," IEEE Transactions on Automatic Control, vol. 50, no. 2, pp. 169-182, 2005.

[14] H. Wang, X. Liao, T. Huang, and C. Li, "Improved weighted average prediction for multi-agent networks," Circuits, Systems and Signal Processing, vol. 33, no. 6, pp. 1721-1736, 2014.

[15] B. Liu, W. Lu, and T. Chen, "Consensus in networks of multiagents with switching topologies modeled as adapted stochastic processes," SIAM Journal on Control and Optimization, vol. 49, no. 1, pp. 227-253, 2011.

[16] H. Li, X. Liao, T. Dong, and L. Xiao, "Second-order consensus seeking in directed networks of multi-agent dynamical systems via generalized linear local interaction protocols," Nonlinear Dynamics, vol. 70, no. 3, pp. 2213-2226, 2012.

[17] L. Xiao and X. Liao, "Periodic intermittent consensus of secondorder agents networks with nonlinear dynamics," International Journal of Control, Automation and Systems, vol. 12, no. 1, pp. 23-28, 2014.

[18] W. Yu, G. Chen, M. Cao, and J. Kurths, "Second-Order consensus for multiagent systems with directed topologies and nonlinear dynamics," IEEE Transactions on Systems, Man, and Cybernetics, Part B: Cybernetics, vol. 40, no. 3, pp. 881-891, 2010.

[19] S. Wang and D. Xie, "Consensus of second-order multi-agent systems via sampled control: undirected fixed topology case," IET Control Theory \& Applications, vol. 6, no. 7, pp. 893-899, 2012.

[20] F. Xiao and L. Wang, "Asynchronous consensus in continuoustime multi-agent systems with switching topology and timevarying delays," IEEE Transactions on Automatic Control, vol. 53, no. 8, pp. 1804-1816, 2008.

[21] Y. Zhang and Y. P. Tian, "Consentability and protocol design of multi-agent systems with stochastic switching topology," Automatica, vol. 45, no. 5, pp. 1195-1201, 2009. 
[22] J. Huang, C. Li, and X. He, "Stabilization of a memristor-based chaotic system by intermittent control and fuzzy processing," International Journal of Control, Automation and Systems, vol. 11, no. 3, pp. 643-647, 2013.

[23] S. Boyd, L. El Ghaoui, E. Feron, and V. Balakrishnan, Linear Matrix Inequalities in System and Control Theory, vol. 15, Society for Industrial Mathematics, 1994.

[24] R. A. Horn and C. R. Johnson, Matrix Analysis, Cambridge University Press, 1985. 


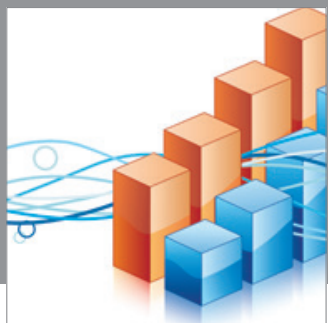

Advances in

Operations Research

mansans

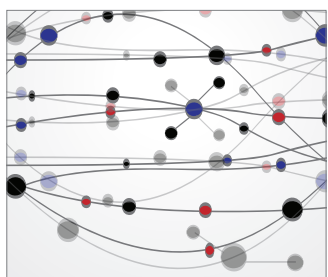

The Scientific World Journal
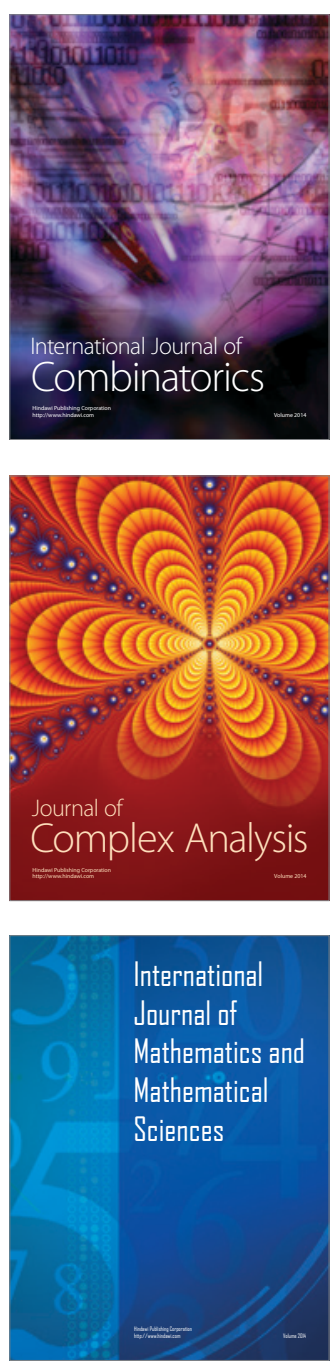
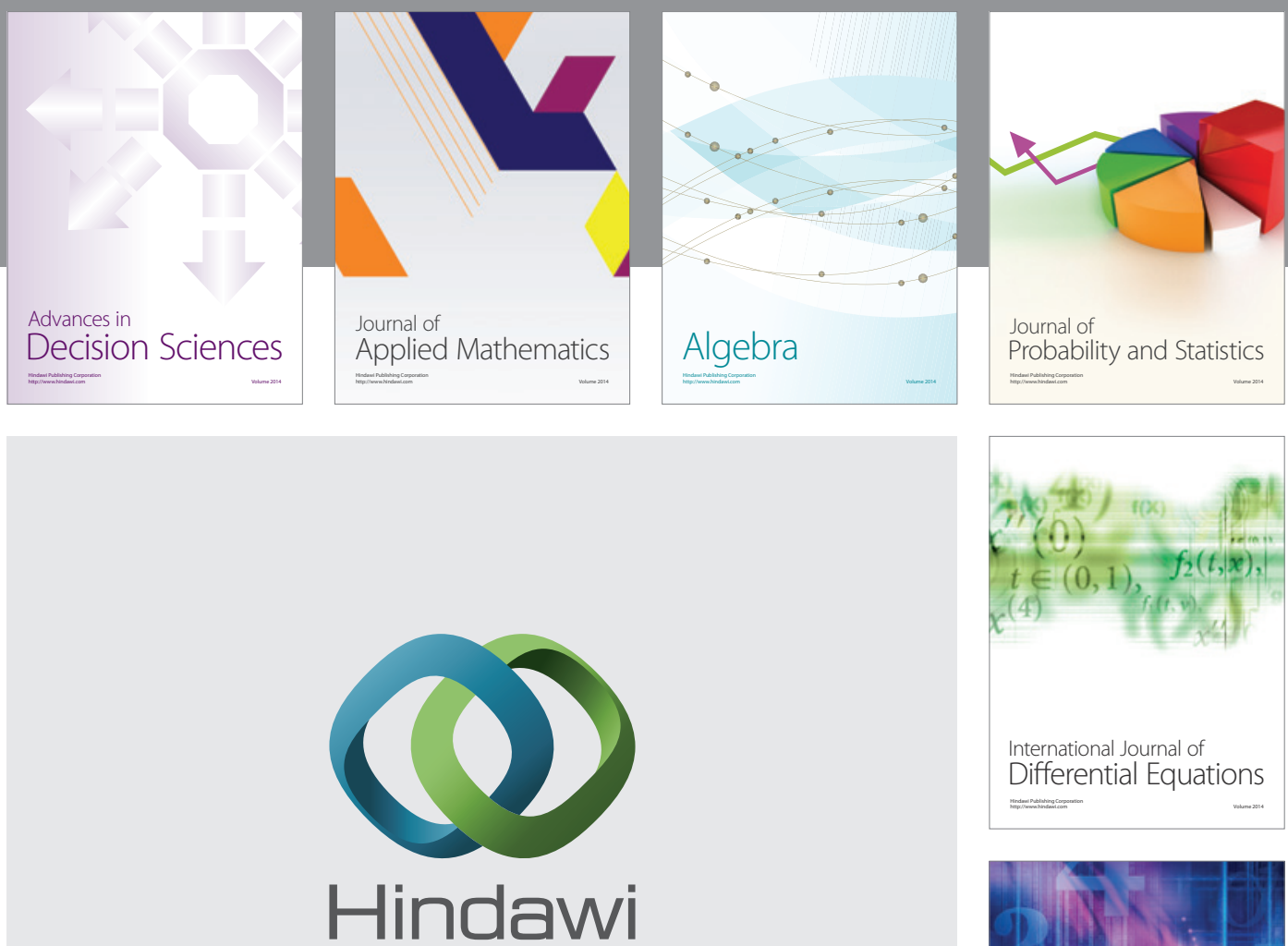

Submit your manuscripts at http://www.hindawi.com
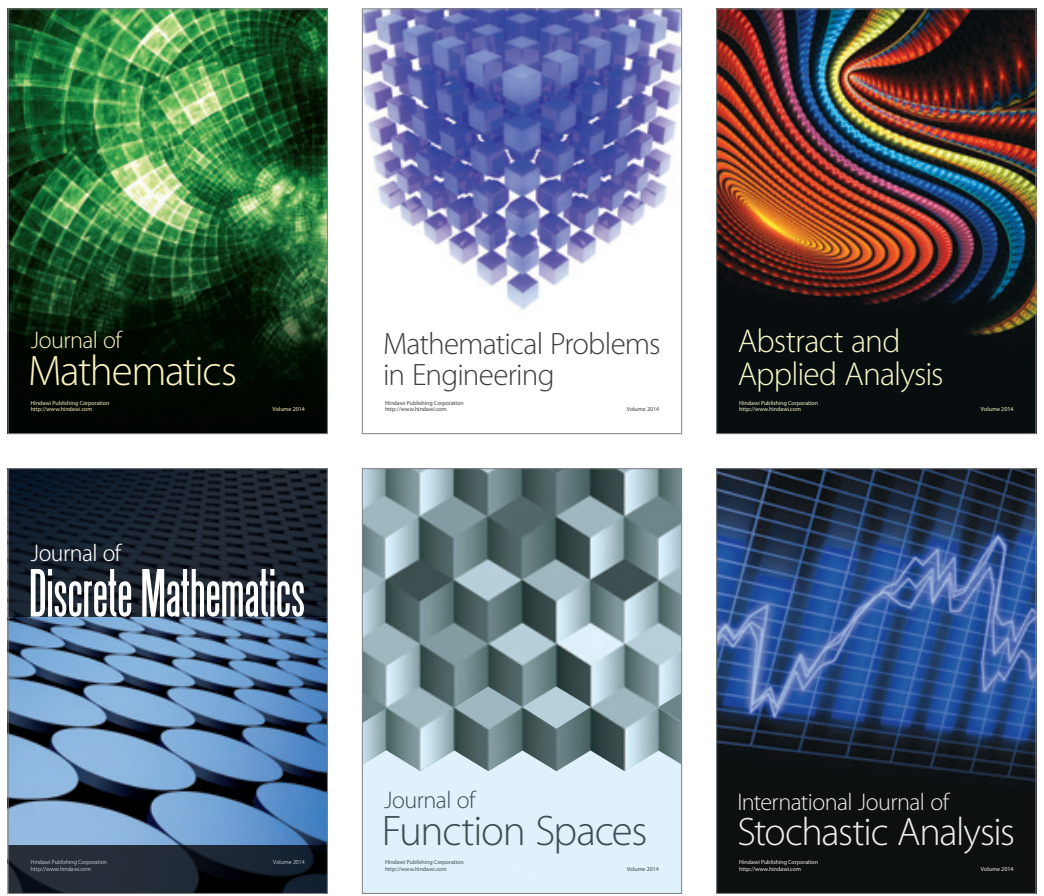

Journal of

Function Spaces

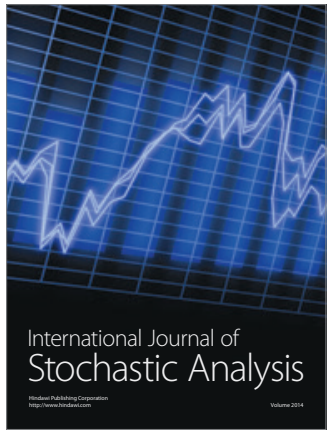

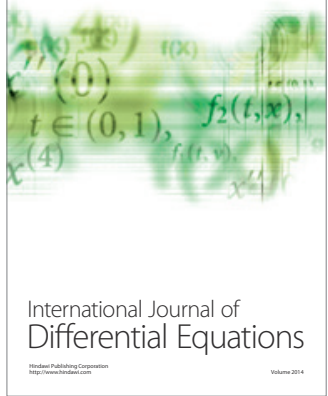
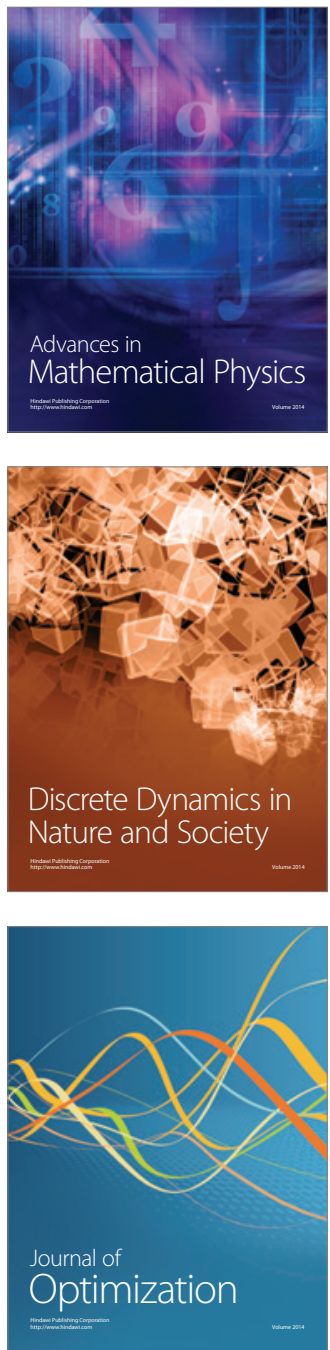